\title{
Integrable Three-Body Systems with Distinct Two-Body Forces
}

\author{
JENS HOPPE and STEFAN THEISEN
}

Instutur fur Theoretist he Physik, Unirersität Karisruhe, PO Box 6.380, D-7500 Karlsruhe. Germany'

(Received 18 March 1991)

\begin{abstract}
Translationally invariant one-dımensional three-body systems with mutually different pair potentials are derived that possess a third constant of motron, both classically and quantum-mechanicaliy; a Lax pair is given, and all (even) regular solutions of the corresponding functional equation ate oblained
\end{abstract}

AMS subject elassifications (1991). 70F05, 70H99

Deriving a functional equation that guarantees the integrability of the so-called Calogero-Moser systems [1], it has been assumed that all particles interact by means of the same two-body force. As a consequence, one finds that this force has to be singular at zero distance. Considering a more general Ansatz for the Lax matrix, however, we were led to a functional equation, which - in the simplest case, i.e. three mutually interacting particles of equal mass - also possesses solutions that are regular (!) at the origin.

Consider the following quantum-mechanical, or classical, Hamiltonian:

$$
H=\frac{1}{2 m}\left(p_{1}^{2}+p_{2}^{2}+p_{3}^{2}\right)+f\left(q_{1}-q_{2}\right)+g\left(q_{2}-q_{3}\right)+h\left(q_{1}-q_{3}\right)
$$

Clearly,

$$
[P, H]=0, \quad P=p_{1}+p_{2}+p_{3} .
$$

Let us then look under which conditions

$$
Q=\frac{1}{3 m}\left(p_{1}^{3}+p_{2}^{3}+p_{3}^{3}\right)+p_{1}(f+h)+p_{2}(f+g)+p_{3}(g+h)
$$

will (Poisson-) commute with $H$ (it automatically commutes with $P$, and for suitably smooth, real $f, g, h$ will also be Hermitean, despite its nonsymmetric form):

$$
\begin{aligned}
{[Q, H]=} & \frac{1}{3 m}\left[p_{1}^{3}+p_{2}^{3}+p_{3}^{3}, f+g+h\right]+ \\
& +\frac{1}{2 m}\left[p_{1}(f+h)+p_{2}(f+g)+p_{3}(g+h), \mathbf{p}^{2}\right]+ \\
& +\left[p_{1}(f+h)+p_{2}(f+g)+p_{3}(g+h), f+g+h\right] .
\end{aligned}
$$


The first and second terms cancel identically, while the condition that the third term should be zero, reads

$$
\begin{aligned}
& h(x+y)\left(f^{\prime}(x)-g^{\prime}(y)\right)+ \\
& \quad+h^{\prime}(x+y)(f(x)-g(y))+ \\
& \quad+f(x) g^{\prime}(y)-f^{\prime}(x) g(y)=0
\end{aligned}
$$

where we have put $x=q_{1}-q_{2}$ and $y=q_{2}-q_{3}$. Note that if $f=(f, g, h)$ solves (5), so will

$$
\mathbf{f}+(c, c, c), \quad c \cdot \mathbf{f}, \quad \mathbf{f}(c \cdot), \quad c \in \mathbb{R}
$$

as well as permuting $f$ and $g$.

We would like to solve (5) with the following two assumptions ${ }^{\star}$

$$
\begin{aligned}
& f(0), \quad g(0), \quad h(0) \text { finite, } \\
& f^{\prime}(0)=g^{\prime}(0)=h^{\prime}(0)=0 .
\end{aligned}
$$

Setting in (5) $y=0$ or $x=0$, respectively, we get

$$
\begin{aligned}
& f(x)=g(0)\left(1+\frac{g(0)-f(0)}{h(x)-g(0)}\right), \\
& g(y)=f(0)\left(1+\frac{f(0)-g(0)}{h(y)-f(0)}\right)
\end{aligned}
$$

as the solutions of the resulting ordinary differential equations; we have thereby used the first symmetry in (6) to set

$$
h(0)=0 .
$$

Also, we assume $f(0), g(0)$, and $f(0)-g(0)$ to be different from zero (otherwise the solutions reduce to those of an effective two-body problem). In any case, (8) implies that

$$
f(x) \cdot g(x)=f(0) \cdot g(0)
$$

Substituting in (5) $g$ and $h$ in terms of $f$, yields

$$
\begin{aligned}
& (f(x+y)-f(0))(f(x+y)-g(0))\left(f^{\prime}(x)+f(0) g(0) \frac{f^{\prime \prime}(y)}{f^{2}(y)}\right)+ \\
& +(f(0)-g(0)) f^{\prime}(x+y)\left(f(x)-\frac{f(0) g(0)}{f(y)}\right)- \\
& -f(0)(f(x+y)-g(0))^{2}\left(\frac{f^{\prime}(x)}{f(y)}+\frac{f(x) f^{\prime}(y)}{f^{2}(y)}\right)=0 .
\end{aligned}
$$

* Thus, excluding the (regular) 'Toda solutions' $f(x)=f_{0} \mathrm{e}^{a x}, g(y)=g_{0} \mathrm{e}^{a y}, h(z)=h_{0} \mathrm{e}^{-a z}$, as well as the (singular) 'Calogero-Moser solutions' $f=g=h=\mathscr{P}(x)$. 
Expanding (11) around $y=0$, and using the remaining symmetries in (6) to put, without loss of generality,

$$
f(0)=1, \quad f^{\prime \prime}(0)= \pm 2(g(0)-1) .
$$

we find (as a necessary, but a-priori not sufficient condition) that the following ordinary differential euqation has to be satisfied by $F(x):=f(x)-g(0)$

$$
F^{\prime \prime} F-F^{2}=2 \varepsilon F\left(t(t-1)-F^{2}\right), \quad t:=g(0), \quad \varepsilon= \pm 1
$$

which can easily be seen to imply

$$
F^{\prime 2}=-4 \varepsilon\left(F^{3}+(2 t-1) F^{2}+t(t-1) F\right)
$$

or, letting $H:=-\mathrm{E}(F+(2 t-1) / 3)$,

$$
\begin{aligned}
& H^{\prime 2}=4 H^{3}-g_{2} H-g_{3}, \\
& g_{2}=\frac{4}{3}\left(t^{2}-t+1\right) \\
& g_{3}=-\frac{88}{27}(t+1)(t-2)\left(t-\frac{1}{2}\right) .
\end{aligned}
$$

As is well known, (15) may be taken as the defining equation for the Weierstrass $\mathscr{P}$-function [2]. Thus

$$
H(x)=\mathscr{P}\left(x+x_{0}\right)
$$

The discriminant $\Delta=g_{2}^{3}-27 g_{3}^{2}$ turns out to be

$$
\Delta=16 t^{2}(1-t)^{2}>0,
$$

so that the two half-periods $\omega_{1}$ and $\omega_{2}$ are real and purely imaginary, respectively [2]. Writing

$$
H^{\prime 2}=4\left(H-e_{1}\right)\left(H-e_{2}\right)\left(H-e_{3}\right),
$$

we find that $H^{\prime}(0)=0$ implies that $x_{0}$ is one of the half-periods $\omega_{1}, \omega_{2}$, or $\omega_{1}+\omega_{2}$, as $\mathscr{P}$ (which is a single-valued doubly-periodic function) takes the values $e_{1}, e_{3}$ and $e_{2}$, at these points, respectively $[2,(8.163)]$.

Thus, $H$ is even and the scaling symmetry in (6) can actually be extended to purely imaginary $c$, which allows us to choose

$$
\varepsilon=-1
$$

without loss of generality. Calculating the $e_{1}$ (letting $e_{1}>e_{2}>e_{3}$, following the notation of [2]), we find, e.g., for $0<t<1$

$$
e_{1}=\frac{2-t}{3}, \quad e_{2}=\frac{2 t-1}{3}, \quad e_{3}=-\frac{1}{3}(t+1) .
$$

Thus, $H(0)=e_{1}$ (cf. (12)), and $x_{0}=\omega_{1}$; hence,

$$
f(x)=\mathscr{P}\left(x+\omega_{1}\right)-e_{3} .
$$


Using (8), and special cases of the addition theorem $[2,(8.166,2)]$ for $\mathscr{P}(u+v)$, (20a) implies

$$
\begin{aligned}
& g(x)=\mathscr{P}\left(x+\omega_{1}+\omega_{2}\right)-e_{3}, \\
& h(x)=\mathscr{P}\left(x+\omega_{2}\right)-e_{3} .
\end{aligned}
$$

Note that all three functions are $\geqslant 0$ and that (20), when put into (1), corresponds to two particles ( 1 and 2 ) tightly bound together, while the third interacts via oscillatory potentials.

We can (and need to) check, then, that (20) indeed satisfies (5) - just use the addition theorem for $\mathscr{P}\left(\left(x+\omega_{1}\right)+\left(y+\omega_{1}+\omega_{2}\right)\right)$. Applying (6) to (20) - including imaginary scale transformations - finally yields the general (modulo permutations of $f, g$, and $h$ ) solution of (5) subject to (7). Looking at how (20) satisfies (5), we are easily led to

$$
\begin{aligned}
& f(x)=\mathscr{P}\left(x+\omega_{1}+a\right) \\
& g(x)=\mathscr{P}\left(x+\omega_{1}+\omega_{2}+b\right) \\
& h(x)=\mathscr{P}\left(x+\omega_{2}+a+b\right) \quad a, b \in \mathbb{R}
\end{aligned}
$$

satisfying (5) (but, in general, not (7), of course).

Naturally, we would like to know how $f, g$; and $h$ look, written as power series. Instead of giving the Taylor expansions of $\mathscr{P}$ around, its half-periods in their standard form [3], we would like to present them in the form which we had originally deduced from (5), by making a power series Ansatz (and comparing low powers of $x$ and $y$ ), before we obtained (20) in closed form:

$$
\begin{aligned}
& f(x)=1+(t-1) \sum_{1}^{\infty} A_{m} \quad 1(t)(-)^{m} x^{2 m}, \\
& g(x)=t\left(1+(t-1) \sum_{1}^{\infty} B_{m-1}(t)(-)^{m} x^{2 m}\right), \\
& h(x)=-t \sum_{1}^{\infty} C_{m-1}(t)(-)^{m} x^{2 m}
\end{aligned}
$$

$A_{m}, B_{m}$, and $C_{m}$ are $m$ th order polynomials (in $t$ ) satisfying various identities and recursion formulae, such as

$$
\begin{aligned}
A_{m}(t)= & (-)^{m} C_{m}(1-t), \quad B_{m}(t)=-t^{m}(-)^{m} C_{m}\left(1-\frac{1}{t}\right), \\
C_{m}(t)= & \frac{1}{(m+1)(2 m+1)}\left[t C_{m-1}(t)+(-)^{m-1}(t-1) C_{m-1}(1-t)\right]+ \\
& +\sum_{t=1}^{m}\left(\frac{m+1-j}{m+1}\right)(-)^{t-1} C_{j-1}(1-t) C_{m-j}(t), \quad C_{0}=1 .
\end{aligned}
$$




$$
\begin{aligned}
& C_{m}=A_{m}+\sum_{1}^{m} A_{j}, C_{m},=-B_{m}-t \sum_{1}^{m} B_{t-1} C_{m,}, \\
& C_{m}(l)=t^{m} C_{m}\left(\frac{1}{t}\right),
\end{aligned}
$$

to list a few of them.

Using (23), we find

$$
\begin{aligned}
& A_{0}=C_{11}=1, \quad B_{0}=-1, \\
& A_{1}=\frac{1}{3}(t-2), \quad C_{1}=\frac{1}{3}(t+1), \quad B_{1}=\frac{1}{3}(2 t-1), \\
& A_{2}=\frac{1}{45}\left(2 t^{2}-17 t+17\right), \quad B_{2}=-\frac{1}{45}\left(17 t^{2}-17 t+2\right) . \\
& C_{2}=\frac{1}{45}\left(2 t^{2}+13 t+2\right), \\
& C_{3}=\frac{1}{3 \cdot 3 \cdot 5 \cdot 7}\left(t^{3}+30 t^{2}+30 t+1\right) .
\end{aligned}
$$

Concerning the general expression for $C_{m}$, partial results like

$$
\begin{aligned}
& C_{m}(0)=\frac{2^{2 m+1}}{(2 m+2) !}, \quad \frac{C_{m}^{\prime}(0)}{C_{m}(0)}=-\frac{m}{2}+\frac{1}{2}\left(2^{2 m}-1\right), \\
& C_{m}(1)=2^{2(m+2)} \sum_{n=1}^{m+1} b_{2 n} b_{2(m+2-n)} \frac{\left(2^{2 n}-1\right)\left(2^{2(m+2-n)}-1\right)}{(2 n) !(2(m+2-n)) !} \\
& b_{n}=\mid \text { Bernoulli numbers } \mid[2],
\end{aligned}
$$

and the form of the expansion found in [3] indicates that a manageable closed expression for $C_{m}(t)$ may be difficult to find.

Let us now give a Lax pair for the classical systems described by (1), with $f, g, h$ as given in (20):

$$
\begin{aligned}
& m=1, \quad 0<t<1, \quad f_{i 3}=f_{t 3}\left(q_{t}-q_{j}\right)=-f_{p} . \\
& L=\left(\begin{array}{ccc}
p_{1} & i f_{12} & i f_{13} \\
-i f_{12} & p_{2} & i f_{23} \\
-i f_{13} & -i f_{23} & p_{3}
\end{array}\right), \quad M=\left(\begin{array}{ccc}
z_{1} & f_{12}^{\prime} & f_{13}^{\prime} \\
f_{12}^{\prime} & z_{2} & f_{23}^{\prime} \\
f_{13}^{\prime} & f_{23}^{\prime} & z_{3}
\end{array}\right), \quad i \dot{L}=[M, L], \\
& f_{12}=\left(\operatorname{sn}\left(x+\omega_{1}, \sqrt{t}\right)\right)^{-1}, \\
& f_{23}=\left(\operatorname{sn}\left(x+\omega_{1}+\omega_{2}, \sqrt{t}\right)\right)^{-1}, \\
& f_{13}=\left(\operatorname{sn}\left(x+\omega_{2}, \sqrt{t}\right)\right)^{-1} .
\end{aligned}
$$

where the $z$, have to satisfy

$$
\begin{aligned}
& \left(z_{1}-z_{2}\right) f_{12}=f_{23} f_{13}^{\prime}+f_{23}^{\prime} f_{13}, \\
& \left(z_{2}-z_{3}\right) f_{23}=-f_{12} f_{13}^{\prime}-f_{12}^{\prime} f_{13}, \\
& \left(z_{1}-z_{3}\right) f_{17}=f_{12} f_{23}^{\prime}-f_{12}^{\prime} f_{23} .
\end{aligned}
$$


We can show that the $z$, may be taken to be of the form

$$
z_{i}= \pm \sum_{j} \frac{f_{i j}^{\prime \prime}}{2 f_{i j}^{\prime}}
$$

The consistency condition for (27), however, yields (5), with $f=f_{12}^{2}, g=f_{23}^{2}$, and $h=f_{13}^{2}$.

Let us conclude by noting that our results extend to the case of $N=N_{1}+N_{2}+N_{3}$ particles, where (up to (6)) particles of equal type interact via $\mathscr{P}(x)$ while particles of type $(1,2),(1,3),(2,3)$ interact via $\mathscr{P}\left(x+\omega_{1}\right), \mathscr{P}\left(x+\omega_{2}\right)$, $\mathscr{P}\left(x+\omega_{1}+\omega_{2}\right)$, respectively.

\section{Acknowledgement}

We would like to thank $M$. Bordemann, O. Eyal, W. Lang, O. Ogievetsky, and A. M. Perelomov for useful discussions.

\section{References}

1. Moser, J., Adv. Math. 16, 197 (1975);

Calogero, F., Lett. Nuovo Cimento 13, 411 (1975)

Oishanetsky, M. A. and Perelomov, A. M., Invent. Math. 37, 93 (1976).

2. Gradshteyn, I. S. and Ryzhik, I. M., Tables of Integrals Series and Products, Academic Press, New York, 1965

3. Abramowitz, M. and Stegun. I. (eds), Handbook of Mathematical Functions, National Bureau of Standards, 1964.

Note added: While this paper was in press, Martin Bordemann pointed out to us that $H$ (as in (1), with $(20)-(21)$ ) is actually canonically equivalent to a model indicated in the second reference of [1]. 